\title{
Pobreza y Participación
}

\author{
Alejandro Natal \\ Facultad de Ciencias Politicas y Administración Pública UAEM
}

\section{Introducción}

A partir de los años sesenta en diversas regiones del mundo se comenzaron a implementar diversos programas de desarrollo social que bajo un esquema similar intentaban solucionar los problemas de los pobres del campo a través de modelos de satisfacción de necesidades básicas y de participación de los propios beneficiarios. Sin embargo, a la vuelta de treinta años nos encontramos con que muchos de esos programas no han arrojado los resultados esperados. Algunos incluyeron en sus diseños una "participación" que no pasó de ser retórica y sus resultados fueron burbujas populistas que desgastaron a los participantes y sólo dispendiaron recursos. Otros programas que con diseños más creativos buscaban resolver situaciones de pobreza a través de la participación de los beneficiarios fallaron en gran medida también.

Mi tesis es que entre las principales razones que hacen fracasar muchos de los programas de desarrollo rural se encuentran Particularmente, primero en la falta de desagregación de la pobreza, el desconocimiento - o la generalización- sobre sus diferentes causas y tipología que llevan a que los programas atiendan la periferia de los problemas sin llegar nunca al corazón de los mismos. Y por otro lado en la falta de envolvimiento real de las poblaciones beneficiadas lo que reduce los programas a meros "diálogos de sordos" en donde la burocracia planea programas que la gente del campo no comparte de fondo. Este planteamiento se puede reducir a dos premisas: (a) el que muchos programas combaten síntomas de la pobreza y no sus causas y (b) a que no involucran activa y críticamente a las poblaciones que intentan beneficiar.

A partir de estas dos premisas intento esbozar ideas para iniciar debate e investigación sobre dos preguntas que me parecen esencialmente relevantes. La primera se referiría al Cómo se identifican los problemas o necesidades a combatir (¿cómo saber porque los pobres son pobres?), y la segunda el cómo se puede atacar ese problema de manera conjunta - con los pobres mismos (¿cómo pueden los pobres dejar ellos mismos de serlo?). 
Este ensayo no pretende llegar a conclusiones definitivas, sino incorporar a la discusión algunos elementos que a mi juicio han quedado olvidados y que me parecen claves para enfrentar el problema de la pobreza.

\section{1. ¿Cómo se identifican los problemas o necesidades a combatir?}

Ideas para análisis del proceso de combate a la pobreza.

Primeramente, para efectos de este análisis quiero presentar los que para mí son los diferentes "momentos", o etapas, en el proceso de combate a la pobreza. Aunque aquí los presento de manera separada, los entiendo vinculados entre sí y sólo los clasifico para uso analítico.

Un primer momento es el de la identificación de una situación de pobreza. El segundo es el de organización de la acción, desde arriba o desde abajo, para combatir esta situación de pobreza. En esta etapa se elabora una política pública o se organizan los pobres mismos para la acción colectiva. Un tercer momento es el de la implementación de la acción, programas o proyectos y finalmente, un cuarto momento, es el de la repercusión de la política pública en la vida cotidiana de los beneficiarios.

Para términos de este ensayo, es decir para analizar el cómo se identifican los problemas o necesidades a combatir tenemos que enfocamos particularmente al primer momento, el de identificación. Es en la etapa de identificación, cuando se decide cuál es el problema a resolver o la necesidad a atender. A partir de lo que es entendido por problema o necesidad se organiza la acción e implementación. La acción guarda una relación directa con el proceso de identificación, dependiendo de qué y cómo es identificado el problema o necesidad, se desarrolla una acción específica. El proceso de identificación es una etapa altamente compleja que condiciona la implementación y acción de cualquier programa o proyecto de combate a la pobreza. Sin embargo aún es poco lo que conocemos sobre él. Es por ello fundamental estudiar cómo se identifica una situación de pobreza y la problemática que rodea a este momento de identificación. En la siguiente parte de este ensayo me propongo bocetar algunos elementos que espero contribuyan a sentar las bases para esta discusión.

\section{Algunas reflexiones sobre el proceso de identificación}

\section{Tipos de identificación}

Aunque con algunas variantes, en general en el combate de pobreza se han seguido dos maneras de identificar una situación de pobreza. El más 
generalizado es el de detección. En este modelo las políticas sociales de combate a la pobreza son el resultado de un problema que se ha detectado. Sea la burocracia o algún político en campaña "detecta" un problema que necesita solución. Este problema puede ser vivienda, agua, drenaje, transporte, etc. A partir de esta "identificación desde arriba" se elabora un programa para resolverlo con lo que entramos en el segundo momento, el de organización para la acción.

Un segundo modelo de identificación es el de autodetección. En este modelo son los pobres quienes se organizan por ellos mismos quienes se reúnen para detectar una situación de pobreza. Es supuesto que conociendo ellos sus necesidades y sus problemas se organicen para resolverlos. Ésta es una "identificación desde abajo" a partir de la cual los pobres se organizan para gestionar, o realizar ellos mismos las actividades necesarias (presión política o implementación de servicios) para satisfacer las necesidades que han identificado.

Ambos modelos tienen ventajas y limitaciones y pueden funcionar mejor o peor dependiendo de la situación de pobreza que se enfrente como discutiremos más adelante.

\section{Problemas de la identificación}

En el proceso de identificación, bajo cualquiera de los modelos anteriores, hay varios grupos de problemas que complejizan el cómo saber discernir entre síntomas y causas de la pobreza. Entre los más importantes tenemos: (a) que en el mismo proceso de identificación hay limitaciones propias de la estructura y modus de la organización identificadora.; (b) cuestiones ideológicas y culturales de las organizaciones que identifican la pobreza de otros o su propia pobreza.; (c) que las situaciones de pobreza son diferentes unas de otras así como la manera en la que sus diferentes síntomas se manifiestan; y finalmente $(\mathrm{d})$ que los problemas de pobreza son sentidos de diferente manera por distintos grupos de pobres.

Para efectos de este ensayo considero particularmente importante presentar algunas reflexiones iniciales para analizar más profundamente cada uno de estos puntos. En el siguiente apartado presentaré algunos elementos que me parecen necesarios para ir ubicando los problemas del modus de las organizaciones identificadoras, las diferentes maneras en las que se percibe la pobreza, la tipología de la pobreza y las diferencias en vulnerabilidad. Me propongo esbozar algunos elementos que nos permitan ir construyendo un modelo para identificar los diferentes problemas que complejizan la identificación de situaciones de pobreza. 


\section{Problemas del modus de la organización identificadora}

En el primer modelo de identificación, el de detección, la responsabilidad de combatir la pobreza es de los burócratas o de algún comité. Está basado en la detección de un problema. La lógica de la identificación por detección es la de ubicar un fenómeno concreto al cuál se le pueda, de preferencia, aplicar alguno de los programas ya existentes o que esté dentro de los campos de acción de la dependencia responsable de la acción. De tal manera que la identificación desde arriba es en general un acomodamiento de situaciones en un padrón identificable para la burocracia. No se analiza cada situación de pobreza en particular sino que la identificación es realizada a través de formas administrativas predeterminadas. Esto repercute en el segundo momento de combate a la pobreza, organización para la acción, en donde la solución que se busca intenta solamente acabar con el problema particular que se ataca (vivienda, educación, transporte), y no necesariamente con las causas que le dan origen. Por su naturaleza, vertical desde arriba, sus fines se orientan más a los tiempos y requerimientos de la burocracia que al combate de la pobreza en sí mismo. Su lógica es reportar sobre la solución del problema y no hacer seguimiento sobre las consecuencias de la acción.

El segundo modelo, en el que los mismos pobres identifican sus necesidades, esta basado en la satisfacción de una necesidad. La lógica de la identificación por auto identificación responde, primero, a cómo perciben los pobres su pobreza y a la manera en que categorizan sus necesidades y segundo a la microeconomía política de los grupos de ciudadanos que se organizan para auto identificar tales necesidades. Respecto a lo primero, la percepción de la propia pobreza y la clasificación de las necesidades tienen que ver con los parámetros culturales de lo que son "las necesidades", con las expectativas de desarrollo del grupo y con sus frustraciones y traumas sociales. Respecto a lo segundo, la microeconomía política, es evidente que en proceso de auto identificación los líderes del grupo tienen un papel decisivo en lo que se identifica como necesidad. Es bien sabido que en estos procesos las necesidades que se identifican no son necesariamente las de los más pobres, sino que responden a los intereses de los líderes, caciques o elites del campo que son los que generalmente organizan a los pobres. En general en este modelo los más pobres no se benefician con este tipo de programas porque -precisamente porque son pobres- no tienen las capacidades organizativas necesarias.

En ninguno de los dos modelos, detección o auto identificación, hay un análisis riguroso del problema en sí mismo. En ninguno de los casos se sabe si se combaten problemas o síntomas. En ambos no se entiende a la pobreza 
como un fenómeno integral. Se busca la solución a un problema concreto (desvinculado de su macro- realidad) o la satisfacción de una necesidad también específica (y divorciada de las razones que originan esa necesidad) y esta solución depende más que a las causas a los modus de la organización identificadora.

(b) La ideologización de la percepción y de la acción contra la pobreza

El cómo se percibe el problema de la pobreza es uno de los problemas fundamentales para su atención. Cualquiera de los actores ya sea la burocracia, ONGs o los pobres tienen un enfoque particular del problema basado en su posición ideológica.

En general - a pesar del supuesto fin de las ideologías-la pobreza se sigue enfocando desde dos grandes perspectivas antagónicas, la residualista y la relacionista. La primera, asume que los pobres han quedado fuera de los beneficios, y por tanto se tiene que "hacer algo" para integrarlos (Bernstein, 1992) al desarrollo -lo que en la práctica quiere decir: integrarlos más rápidamente al mercado (perspectiva seguida e impulsada por el Banco Mundial). La pobreza vista como un problema relacional entiende que los pobres son pobres como consecuencia de las estructuras de producción que reproducen su pobreza. La pobreza se ve entonces como un fenómeno estructural.

A mi juicio ambas perspectivas son limitadas en su potencial analítico y en su capacidad de generar modelos y/o políticas sociales que nos permitan realmente combatir el problema de la pobreza.

Hacia una tipología de la pobreza

En el área en particular del combate a la pobreza es necesario entender a esta como un fenómeno multicausal, donde procesos relaciones y estructuras socioeconómicas se enmarcan y reproducen. La pobreza tiene muchas formas, tales como, no tener tierra, tener bajos salarios, falta de voz política, vulnerabilidad, imperfecciones en el mercado, mal diseño de políticas sociales o de servicios básicos, etc.

En este ensayo quiero proponer una tipología básica y general a partir de la cual podamos construir un modelo para una análisis más específico de las diferentes situaciones de pobreza y su identificación.

1. Un primer tipo de pobreza es aquélla que es resultado de la relación del hombre y su ambiente social. Este tipo de pobreza es el resultado de las relaciones de producción y procesos que generan falta de recursos y bajos salarios. 
2. Un segundo tipo de pobreza es el resultado de la relación del hombre con su medio ambiente. Es consecuencia del exceso poblacional y de la presión que la población ejerce sobre los recursos que tiene a su alcance (una severa división de activos) y al medio ambiente. Entre más pobre se es, más se explotan los propios recursos (y los del medio ambiente). Esta "tragedia de los comunes" condena a los pobres a acabar de manera sistemática con cada uno de sus bienes. Cuando esto sucede en un ambiente cambiante, por ejemplo, sujeto a la dinámica del mercado, los pobres del campo pueden adoptar formas no sostenible s y no "racionales" de explotación. La racionalidad de explotación que opera entonces es la racionalidad de la desesperanza.

3. Finalmente existe una pobreza que es el resultado de la relación del hombre con los imprevistos. Ésta es una pobreza "traumática" que brota de manera "espontánea" en tiempo y espacio, y que es el resultado de desastres ambientales o humanos tales como inundaciones, guerras, etc.

Cada tipo de pobreza implica un tipo de "vulnerabilidad" y tiene ciertos elementos que la reproducen y refuerzan. Cada tipo de vulnerabilidad produce diferentes formas de reproducción de pobreza. Esas formas podrían imaginarse como diferentes curvas espirales que van impactando a diferentes grupos de la población, afectando en general más a los más pobres.

¿Quiénes son los pobres en el campo?

Es importante que estemos alerta para el evitar caer en la perspectiva esterotipada que ve a los pobres del campo como una masa de gente pobre y desamparada, como las víctimas del capitalismo urbano. Los pobres del campo están altamente diferenciados no sólo en actividades e ingresos sino también en expectativas, necesidades potencialidades. Robert Chambers (1998a: 6-8) señala lo deshumanizante que es mirar a los pobres del campo como una agregado estadístico y enfatiza en la importancia de desagregar a los pobres identificando quienes son pobres en el campo y quienes no, pero sobre todo estudiando por qué son pobres los que lo son.

La primera distinción la podríamos hacer entre los pobres y los extremamente pobres. Esto es atender a la distribución de la pobreza bajo la línea de pobreza. Lo que nos permite relacionar la brecha entre las pobrezas con las líneas de pobreza que se encuentren y entender mejor como diferentes grupos de pobres experimentan la pobreza de diferente manera. De un estudio de este tipo podríamos concluir patrones de comportamiento y supervivencia que permitirían ayudar mejor a cada grupo. 
Un segundo elemento sería atender a la "exclusión" de la que son víctimas ciertos grupos. Esta exclusión puede ser una cuestión de género, raza, religión, edad, etc. y puede ir de cuestiones de alimentación y consumo hasta seguridad, educación y mortalidad.

Un tercer elemento es la localización espacial de los pobres al interior de una región o comunidad.

Otro elemento lo constituyen el conjunto de actividades que realizan los pobres para sobrevivir. Diferentes conjuntos de actividades garantizan en ciertos medios mayor o menor vulnerabilidad que otros. Hay pobres que concentran sus esfuerzos en una sola actividad de la cual dependen exclusivamente para su supervivencia. Por otro lado hay pobres que diversifican y realizan muchas pequeñas actividades en las que -en conjunto- sustentan su ingreso.

Los "pobres de una actividad" se encuentran totalmente dependientes de ella para su economía. Pueden realizar sólo esa actividad porque han encontrado que es la que mejor resuelve sus necesidades, por falta de tierra, o de experiencia o incluso por problemas estructurales.

Ninguno de los dos tipos es mejor, sino que dependen de las condiciones específicas de la región o comunidad en la que viven, tales como tierra y clima, condiciones de producción, salud, acceso a mercados, etc.

De lo anteriormente expuesto se deduce que la pobreza no es la misma en todos los contextos, que es percibida diferente, tiene distintas causales, y sobre todo se reproduce de diversas maneras entre diferentes grupos de pobres. Muchos programas de atención y desarrollo dirigidos a los pobres fracasan simplemente porque han desatendido a las limitaciones de sus propios procesos, a las diferencias de percepción, y a las diferencias entre tipos pobreza y vulnerabilidad. Cuando el combate a la pobreza tiene problemas serios de identificación, los programas se convierten en "palos de ciego" que benefician por casualidad y sin sustentabilidad.

Las políticas públicas tienen que tener suficiente flexibilidad y dinamismo para ser capaces de adaptarse y responder a los diferentes tipos de pobreza a los que se enfrentan. Pero, si queremos efectividad, esta flexibilidad y dinamismo no pueden ser resultado de la improvisación. Es claro que las causas de la pobreza no pueden ser identificadas a priori. Pobreza es un fenómeno multidimensional que requiere un análisis cuidadoso e interdisciplinario. Muchas veces las causas de la pobreza están escondidas en una multiplicidad compleja de acciones y relaciones culturales y económicas difíciles de desenmarañar. El mapa de la pobreza de un país es el resultado de complementariedades y falta de relaciones 
entre factores culturales, sociológicos, económicos y políticos. El análisis detallado de las limitaciones culturales, del contexto socio- económico y de la dinámica político-social en la que se insertan y persisten las situaciones de pobreza y exclusión es fundamental para un combate efectivo de la pobreza. Si se quiere que las políticas de alivio tengan impacto real en el desarrollo de un país, es necesario reorganizar el marco institucional en el que ésta se combate, pero particularmente es necesario crear mecanismos para analizarla adecuadamente, estudiar sus cambios y diseñar programas sostenibles que reduzcan los riesgos y la vulnerabilidad de los pobres. Un combate adecuado implicaría mayor conocimiento de la situación local que se intenta resolver. Implica incrementar el análisis, la reflexión y el estudio adecuado de (a) los problemas del modus de las organizaciones identificadoras; (b) de la postura ideológica desde la que se mira la pobreza; (c) del tipo de pobreza al que se enfrenta y de las espirales de vulnerabilidad que este engendra y (d) cómo es percibida de diferente manera por diferentes grupos de pobres.

Para ello la participación ciudadana es un factor crítico para entender las dinámicas políticas, económicas, sociales y naturales que interactúan para perpetuar las condiciones de pobreza en cada región en particular.

\section{Visión pragmática de lo que es pobreza}

Mi visión de la pobreza es más pragmática. Para mí la pobreza es: una situación de exclusión social, donde un grupo de individuos vive bajo condiciones que limitan sus capacidades y potencialidades para producir, competir y/o hacerse de bienes y servicios de manera equitativa con el resto de la sociedad en la que viven.

Por lo tanto es claro que en este ensayo se entiende a la pobreza como un término relativo. La pobreza siempre se mide frente a algo que se quiere alcanzar (objetivos, expectativas, etc.).

De lo anterior se deduce qué -para mí- el problema de la pobreza sólo se podrá combatir cuando seamos capaces de detectar las áreas que propician y perpetúan la exclusión social y las ataquemos de raíz.

El alivio de la pobreza es un problema político-económico (desde local hasta internacional) y es una responsabilidad social.

Muchos programas de desarrollo rural han olvidado esta dupla causalidad. No se pueden resolver los problemas de pobreza dejando a los pobres al garete de los vaivenes del mercado ni tampoco se les ayuda mucho supliéndolos eventualmente - o subsidiándolos permanentemente- de algunos satisfactores. 
La pobreza no será erradicada del campo mientras no la analicemos en el marco de estos dos elementos mancornados. No son suficientes los programas de asistencia social sin desarrollo económico y es inútil tratar de hacer llegar créditos al campo sin un programa integral de política social coherente a las necesidades y expectativas de los pobres del campo.

Para cerrar la brecha que separa las capacidades actuales y las potenciales de los pobres se requiere no sólo de mejoras cuantitativas a los factores de producción tradicionales -tierra, trabajo y tecnología, capital y actitud emprendedora-, sino -y sobre todo-, se requiere de atención a los factores cualitativos que reproducen la pobreza en el campo.

\section{Ideas para un modelo alternativo}

Lo primero que se necesita saber es donde están los pobres. Quiénes y dónde están localizados. Necesitamos hacer "mapas -detallados- de la pobreza". Esto es mapas que ubiquen físicamente los varios niveles de pobreza y que nos permitan cruzar esta información con aquéllas relacionadas a servicios públicos, producción agrícola, etnicidad, recurrencia de desastres naturales y deterioro ambiental, transportes, etc. Estos mapas deberán de permitir el registro de cambios en la situación geográfica, social y económica de los pobres. Sólo de esa manera podremos estar en posibilidades de establecer las posibles causas de la pobreza de la región y actuar en consecuencia.

El siguiente paso es diseñar estrategias de participación. En las que los pobres sean capaces de analizar los datos que los "mapas de pobreza" arrojen. Y nos permitan conocer su punto de vista al respecto, así como la manera en que ellos perciben las causas de su pobreza, sus necesidades y vislumbran sus expectativas.

El tercer paso, es diseñar estrategias de participación en el análisis de datos. En donde los pobres sean capaces de analizar los datos que los "mapas de pobreza” arrojen. Y nos permitan conocer su punto de vista al respecto así como la manera en que ellos perciben sus necesidades y vislumbran sus expectativas.

Un cuarto paso es la creación de talleres para la "discusión del desarrollo". Donde los pobres del campo sean enfrentados con sus problemas y se analicen las posibles soluciones a ellos y los costos, beneficios y consecuencias que las diferentes decisiones implicarían.

Finalmente, pero lo más importante, es necesario fomentar la promoción de una participación activa -no retórica- de los pobres en programas del desarrollo. 
Estos últimos puntos: "discusión del desarrollo" y "participación real” las discuto a continuación.

\section{Participación real}

En general cuando hablamos de "participación” entendemos el envolvimiento de los pobres en la provisión de sus propios bienes y servicios.

Recientemente se ha hecho notable que si a los pobres del campo se les da la oportunidad de participar en proyectos que les beneficien, ellos muestran en general mucha disponibilidad para hacerlo. La participación de los pobres del campo aumenta su aceptación y compromiso con los proyectos que son elementos cruciales para el buen desarrollo de un programa.

Un programa de desarrollo que envuelve a la gente provee la base no sólo para mejorar los estándares materiales de bienestar pero también el progreso en su vida social y cultural.

Sin embargo esta "participación" (de la misma manera que la pobreza) se puede ver de muchas diferentes perspectivas, que en general han caído en extremos. Para algunos los pobres no son más que "mano de obra barata" a la que se le tiene qué decir qué hacer (ésta es una perspectiva de derecha que encuentra en la participación un excelente mecanismo para imponer un impuesto a los pobres). Para otros son seres "infalibles" que "saben mejor lo que les con- viene” y que por tanto siempre toman las decisiones más adecuadas (ésta es una perspectiva de la izquierda radical "liberalizante", que busca que fondos públicos sean canalizados a los pobres para que ellos mismos decidan qué hacer con los recursos).

Particularmente no creo en ninguna de las dos opciones. Si los pobres no piensan sobre su pobreza críticamente, no vislumbran sus posibilidades y expectativas no se van a convertir nunca en autores de su desarrollo. Si por otro lado sólo se dedican a pensar opciones sabiendo que éstas van a ser apoyadas -con fondos públicos- no van a desarrollar una conciencia de riesgobeneficio.

Me parece que la participación ciudadana no es en sí misma ni buena ni mala, es una herramienta de la administración del desarrollo y como tal tiene que ser analizada en sus pros y contras en las situaciones concretas en las que se quiera implementarla.

\section{Ventajas de la Participación}

El desarrollo participativo ofrece claramente varias ventajas: Primero, su mayor fuerza radica en la sabiduría y el conocimiento innato que los pobres del 
campo tienen de elementos como por ejemplo el medio ambiente, que les es ínfimamente familiar. Cuando acompañada de una cuidadosa asistencia externa, esta "inteligentsia local" puede resultar en proyectos que son más manejables en alcance y no sólo dependientes en importación tecnológica, que pueden tener menor costo.

Aún más, los pobres mismos, pueden voluntariamente mantener el proyecto una vez que ha sido terminado.

La participación de los pobres aumenta el nivel de innovación y el desarrollo de sistemas tradicionales. Sin embargo, un desarrollo participativo que no esté basado en la capacitación puede tener mucho de participativo pero muy poco de desarrollo.

DP tiene también la ventaja de que bien diseñado ayuda a resolver conflictos individuales e intergrupales.

La participación real de los pobres -y no únicamente en retórica- refuerza la "voz" de los pobres y les da mayor capacidad de negociación frente a grupos más poderosos.

Pero sin duda el mayor punto a favor de este proceso es la independencia que las comunidades rurales pueden adquirir. Si un espíritu de autogestión y cohesión comunitaria logra aparecer como resultado, estos dos factores se convertirán en los pilares de un poder político basado en la comunidad.

Pero este tipo de participación sólo se logra como resultado de un proceso de toma de consciencia crítica sobre los propios problemas. Sólo se genera con bases sólidas en el marco de una "discusión para el desarrollo".

\section{Problemas de la Participación}

Si la participación tiene ventajas claras, es innegable que también tiene desventajas que pueden volverse serias.

Primero, no podemos hablar de participación sin referirla al marco político en el que se desarrolla. Problemas micro-políticos al interior de las propias comunidades como de política regional y nacional pueden impedir un desarrollo de este tipo.

La participación de los pobres tiene muchos costos. En términos de tiempo, horas-hombre y recursos usados para organizar juntas, explicaciones, discusiones y acuerdos.

Los acuerdos nos son siempre las decisiones óptimas, sino -por teoría de juegos- tienden a ser las "segundas mejores", debido a negociaciones entre grupos y sectores. 
Haciendo un balance entre las ventajas y desventajas, resulta claro que si bien las desventajas son importantes son superadas bastante por las ventajas. Si sólo fuera por el hecho de que los pobres desarrollen una capacidad autogestiva sería suficiente.

Un medio que yo propongo para combatir las desventajas es lo que llamo "discusión del desarrollo"

\section{Discusión para el desarrollo}

De entre todos los factores cualitativos que pueden interrumpir la reproducción de la pobreza, la educación es el más importante.

Pero, no me refiero a la educación que hasta ahora han recibido los pobres del campo. En México por fortuna la red de la educación rural, aunque con problemas, es una de las más extendidas del Tercer mundo. Aunque su calidad deja aún mucho que desear.

Me refiero aquí a una educación adecuada que haga que los pobres sean más capaces de evaluar sus intereses, sus objetivos y sus expectativas y sus posibilidades reales para lograr sus objetivos y las consecuencias y costos que eso implica. Una educación que permita a los pobres ser más críticos de sus problemas y de las alternativas de solución a los mismos. Una educación para el desarrollo

Es a través de la educación, la capacitación y el desarrollo de recursos humanos que los pobres pueden ejercer sus derechos políticos y sociales para aprovechar mejor sus oportunidades económicas. El acceso a una mejor educación depende -por otro lado- de la capacidad de negociación política que tengan los pobres y de las dinámicas sociales al interior de sus comunidades.

\section{Educación es poder}

Pero no podemos esperar treinta años a mejorar la educación de los pobres del campo. Tenemos que encontrar sistemas a través de los cuales educación y poder se vayan construyendo a si mismos.

En mi opinión el poder que necesita la gente del campo sólo lo pueden desarrollar ellos mismos a través de la organización de "unidades de desarrollo" basadas en la participación de la gente de cada comunidad en la toma de decisiones y en el planeamiento de su futuro. Estas unidades del desarrollo tienen que ser críticas e reflexivas.

Esto implica que los pobres analicen sus expectativas, sus capacidades y los costos Y los beneficios que esperan recibir del desarrollo. Implica no copiar modelos sino reflexionar sobre los que existen y adaptar o crear fórmulas 
nuevas basadas en el desarrollo institucional de cada comunidad. Esto es lo que yo llamo "discutir el desarrollo".

Lo cual lleva implícito:

1. "Aprender de los pobres";

2. Que los pobres tienen que aprender de ellos mismos y que los pobres del campo pueden aprender de los errores -y aciertos- que nuestra sociedad ha cometido y tratar de evitarlos o adaptarlos a la suya.

3. Los pobres conocen bien su situación y algunas de las causas de su pobreza. Saben mejor como proteger su medio ambiente y solucionar los problemas cotidianos de sus economías. Tienen también mucho que enseñamos en cuanto a actitudes de vida, consumo y ecología.

4. Los pobres son un excelente mecanismo de multiplicación de experiencias. Si estas son compartidas entre ellos -en talleres, encuentros, etc.--

5. Industrialización, masificación urbana, indiferencia y consumismo son también parte del desarrollo capitalista. Tenemos también que compartir esta realidad con los pobres del campo para evitar llevar "las ciudades pérdidas" al campo.

\section{Conclusiones}

La discusión de las "unidades de desarrollo" en cada comunidad debe se ser focalizada, informada y sustentable. Para ello necesitan ser apoyadas por organizaciones con mayor cobertura.

Yo vislumbro una organización intermedia que garantizará la continuación de esfuerzos del desarrollo participativo. Un organismo de representación en el que diferentes grupos -gobierno, ONGs y los pobres -se reúnan continuamente para analizar y discutir estrategias y rumbos de acción. Estos "consejos de desarrollo" podrían estar basados en el principio de "óptimas áreas - económicas, políticas y culturales”. Y deberían ser sujetos a investigaciones periódicas sobre sus finanzas por la Contraloría y firmas independientes de contadores.

Necesitamos institucionalizar el desarrollo participativo, ya que un desarrollo participativo que no sea apoyado por instituciones adecuadas tiende a morir al paso del tiempo. No tiene sustentabilidad.

Precisamos de construir una sociedad más distributiva (Rawls) y benefactora (Boaventura) que involucre a todos los sectores, gobierno, ONGs y los mismos pobres en el proceso. Donde más recursos sean canalizados al campo, pero asegurándose que esos recursos serán usados de manera eficiente y honesta. 
Mucha más atención debe darse al estudio de la antropología, la sociología y la economía política de las zonas rurales en situaciones de pobreza. Es fundamental también desarrollar estudios de desarrollo regionales con particular énfasis al estudio de instituciones, al funcionamiento de las ya existentes y el diseño de nuevas. La administración pública debe dedicar más esfuerzos al estudio de este tipo de situaciones, resaltando sus especificidades en procesos y en sentidos.

Las ciencias de la comunicación probarán ser de inmensa ayuda cuando las enfoquemos, en esta área en particular, al desarrollo rural. El papel de los medios masivos regionales es pieza clave en el desarrollo rural -como lo han demostrado muchos ejemplos.

Para sumarizar, lo que yo estoy proponiendo aquí es:

1. Que la definición de la pobreza es un asunto ideológico.

2. Que la pobreza es poliforme y por tanto se constituye y reproduce de diferente manera en cada contexto en particular.

3. Que por tanto sólo con la participación de todos los actores, especialmente de los pobres, puede ser combatida.

4. Que esta participación tiene que ser lo que yo llamo "participación real", informada, reflexiva y crítica.

5. Estos actores están relacionados en un "área óptima” de desarrollo regional.

6. Que este combate tiene que ser institucional

7. y que tiene que ser crítico.

Las implicaciones de esta propuesta de Desarrollo Participativo Sustentable son cinco:

1. Redistribución del ingreso -hacia una sociedad más justa al estilo de Rawls;

2. Envolvimiento de la sociedad en acciones de combate a la pobreza hacia una sociedad más benefactora al estilo de Boaventura;

3. Un combate analítico -de dos vías- a la pobreza - en el que intento proponer las bases de un modelo para que "desde los pobres" podamos criticar el utilitarismo de Bentham y Stuart Mill- a través de lo que yo llamo "discutir el desarrollo".

4. A través de la discusión del desarrollo llegar a una sociedad más ecológica. Menos desarrollista y más conservacionista.

5. Las macro implicaciones de esta propuesta son el cambio de una democracia representativa a una democracia participativa. 


\section{Bibliografía}

Akerlof, G.A., 1970: “The market of for 'lemons': Quality Uncertainty and the Market Mechanism" in: Quarterly Journal of Economics No.84 (3) Aug.

Arrow, K.J., 1951: Social Choice and Individual values, New York: John Willey.

Bates, R., 1995: "Social Dilemmas and Rational Individuals an Assessment of the New Institutionalism", in: Harris J., Hunter J. and Lewis C., The New Institutional Economics and Third World Development, London and New York:

Contreras and Bennet, 1994: "National Solidarity in the Northem Borderlands: Social Participation and Community Leadership", in: Cornellius A, Craig A, and Fox J" Transforming State-Society Relations in México, San Diego: The National Solidarity Strategy, Center of US-Mexican Studies.

Cornellius, Craig and Fox, 1994: "Transforming State-Society Relations" in México, the National Solidarity Strategy, San Diego Center of US-Mexican Studies.

Korten D. and Alfonso, 1983: Bureaucracy and the Poor.

Korten D, 1980: "Community Organization and Rural Development: a learning process approach" in: Public Administration Review.bre,

Hirschman, O., 1970: Exit, Voice and Loyalty, Cambridge: Harvard.

Knight, A, 1994: "Solidarity: Historical Continuities and Contemporary Implications", in: Cornellius A., Craig A., and Fox J, Transforming State-Society Relations in México, The National Solidarity Strategy, San Diego: Center of US-Mexican Studies

North, D., 1981: Structure and Change in Economic History, New York.

Olson, M., (965: The Logic of Collective Action, London.

Ostrom, E., 1993: Institutional Incentives and Sustainable Development, Westview Press Bolder.

Paul, S., 1987: Community Participation in Development Projects: World Bank Experience, World Bank, Washington D.C.

Paul, S, 1991: Accountability in public services: exit voice and capture, World Bank, Working papers WPS 614, Washington D.C.

Rahman, A., 1993 People's Self-Development, Perspectives on Participatory Action Research, in: Zed Books and University Press Limited, Daka.

Redfield, R., 1956: Peasant Society and Culture, Chicago: University Press

Williamson, O.E., 1985: Markets and Hierarchies, New York: Free Press. 